\title{
PRINCIPALITY OF CURVES ON SANDWICHED SINGULARITIES
}

\author{
JESÚS FERNÁNDEZ-SÁNCHEZ
}

\begin{abstract}
Given a surface $X$ obtained by blowing up a complete $\mathfrak{m}$-primary ideal in the local ring of a point on a non-singular surface $S$, we determine the Picard group of $X$ and the divisor class groups of its singularities. Given a curve $C$ on $X$, we obtain various criteria for $C$ to be locally principal at these singularities. Our criteria are stated in terms of the projection of $C$ onto $S$. A minimal system of local generators of the defining ideal of $C$ is produced, as well as a formula for their number.
\end{abstract}

\section{Introduction}

Sandwiched singularities are normal surface singularities which birationally dominate a non-singular surface. They are rational surface singularities and among them are included all cyclic quotients and minimal surface singularities. Any sandwiched surface singularity can be obtained by blowing up a complete $\mathfrak{m}$-primary ideal in a regular local ring $(R, \mathfrak{m})$. From an algebraic point of view, they are the normal birational extensions of some regular local ring of dimension two. The original interest in sandwiched singularities comes from the problem of how to resolve singularities by using normalized Nash transformations. In [17], Spivakovsky examines sandwiched surface singularities and gives an affirmative answer to this question for the case of surface singularities by studying sandwiched surface singularities. Since then, sandwiched singularities have been studied as a as a useful testing ground for the Nash problem of arcs in $[13,16]$ and from the point of view of deformation theory in $[6]$ and $[10]$.

A sandwiched surface $X$ is a surface obtained by blowing up a complete ideal $I$ in a regular local ring $\mathcal{O}_{S, O}$ of a non-singular surface $S$. In this paper we address the question of the local principality of curves (effective Weil divisors) near the (sandwiched) singularities of $X$. Criteria for the principality of effective divisors on $X$ are given in terms of their projection onto $S$ in relation to the infinitely near base points of $I$. As a consequence, the Picard group of $X$ and the divisor class group of any singularity of $X$ are determined. Our motivation comes from the study of the divisor class group of rational surface singularities in general (see $[14,5]$ ). A criterion for the existence of a local equation defining a given curve near the singularities of $X$ is presented as well. Its interest lies in the fact that it makes it possible to check easily the local principality of curves using the combinatorial properties of the associated Enriques diagrams. We give a procedure for determining minimal sets of generators of the ideals of curves at the singularities of $X$ and provide an easy formula to compute their number. Our approach is based on the point of view of $[9,1]$ and makes use of the theory of infinitely near points as revised and developed in [4].

Received by the editors September 5, 2008.

AMS 2000 Subject Classification 14J17; 32S05; 14C22. 
The paper is organized as follows: concepts and facts about infinitely near points and their connection with sandwiched singularities are reviewed in Section 2. Section 3 deals with the existence of global equations for curves on $X$. The main result is Theorem 3.1, which describes the Picard group of $X$. Then, we provide different criteria to check whether a given curve on $X$ is a Cartier divisor or not. In addition, the divisor class group of any sandwiched singularity is described in Corollary 3.8 . Section 4 is devoted to the technical preparation for Section 5: it introduces a variation of Enriques's unloading procedure. Then, in the last section of the paper and given a curve $C$ on $X$, this procedure gives rise to a weighted cluster $\mathcal{R}_{C}$ from which we are able to check the local principality of $C$ (Theorem 5.3) and obtain a formula for the minimal number of generators of the ideal of $C$ near the singularities of $X$ (Proposition 5.6). Previously, in Proposition 5.2, the sheaf of ideals of any curve $C$ on $X$ is described in terms of the base points of the ideal $I$ blown up to obtain $X$ and the projection of $C$ on $S$. A method to determine a minimal set of generators of these ideals is also given in this section.

\section{Preliminaries and Notation}

Throughout this work the base field is the field $\mathbb{C}$ of complex numbers. A curve will be an effective Weil divisor on a surface. A standard reference for some of the material treated here is the book by Casas-Alvero [4] and the reader is referred to it for definitions and basic facts concerning the theory of clusters of infinitely near points. Let $(R, \mathfrak{m})$ be a regular local two-dimensional $\mathbb{C}$-algebra. Write $S=\operatorname{Spec}(R)$ and $\mathcal{N}_{O}$ for the set of points infinitely near or equal to $O$. A cluster of points of $S$ with origin $O$ is a finite subset $K$ of $\mathcal{N}_{O}$ such that for any $p \in K, K$ contains all points preceding $p$. By assigning integral multiplicities $\nu=\left\{\nu_{p}\right\}$ to the points of $K$, we get a weighted cluster $\mathcal{K}=(K, \nu)$, with the multiplicities $\nu$ called the virtual multiplicities of $\mathcal{K}$. We write $p \rightarrow q$ if $p$ is proximate to $q$. Once an admissible order has been fixed on $K$, we can take $K$ as a set of indices (see $\S 4.3$ of [4]). The proximity relations between the points of $K$ are then codified by means of the proximity matrix $P_{K}$ introduced in [7]. This matrix is a $K \times K$ square matrix defined by taking the entry in the $p$-th row and $q$-th column as

$$
\begin{cases}1 & \text { if } p=q \\ -1 & \text { if } p \text { is proximate to } q \\ 0 & \text { otherwise }\end{cases}
$$

The quantity

$$
\rho_{p}^{\mathcal{K}}=\nu_{p}-\sum_{q \rightarrow p} \nu_{q}
$$

is called the excess of $\mathcal{K}$ at $p$. The cluster $\mathcal{K}$ is consistent if it has no negative excesses. Consistent clusters are characterized as those clusters whose virtual multiplicities are realized effectively by some curve on $S$. If $\mathcal{K}$ is consistent, we say that $p$ is a dicritical point of $\mathcal{K}$ if $\rho_{p}^{\mathcal{K}}>0$ and we write $\mathcal{K}_{+}$and $K_{0}$ for the set of dicritical and non-dicritical points of $\mathcal{K}$, respectively.

Let $\mathbf{I}$ denote the semigroup of complete $\mathfrak{m}$-primary ideals in $R$. If $\mathcal{K}$ is a weighted cluster, the equations of all the curves going through it define an ideal $H_{\mathcal{K}} \in \mathbf{I}$ (see [4] 8.3). Any ideal $J \in \mathbf{I}$ has a cluster of base points associated to it, denoted by $B P(J)$, 
which consists of the points shared by, and the multiplicities of, the curves defined by generic elements of $J$. The maps $J \mapsto B P(J)$ and $\mathcal{K} \mapsto H_{\mathcal{K}}$ are reciprocal bijections between $\mathbf{I}$ and the set of consistent clusters with no points of virtual multiplicity zero (see [4] 8.4.11 for details). If $p \in \mathcal{N}_{O}$, we denote by $I_{p}$ the (simple) ideal generated by the equations of the branches going through $p$ and by $\mathcal{K}(p)$ the weighted cluster corresponding to it under the above bijection. If $\mathcal{K}=(K, \nu)$ is consistent, the excess of $\mathcal{K}$ at $p$ equals the power of $I_{p}$ in the Zariski factorization of $I$. Thus $\mathcal{K}$ can be written in a unique way as

$$
\left.\mathcal{K}=\sum_{p} \rho_{p}^{\mathcal{K}} \mathcal{K}(p) \quad \text { (see 8.4.5 of }[4]\right) .
$$

Otherwise, if $\mathcal{K}$ is not consistent, we have a decomposition of $\mathcal{K}$ as

$$
\mathcal{K}=\mathcal{K}^{+}-\mathcal{K}^{-}
$$

where $\mathcal{K}^{+}=\sum_{p} \rho_{p}^{+} \mathcal{K}(p)$ and $\mathcal{K}^{-}=\sum_{p} \rho_{p}^{-} \mathcal{K}(p)$ are consistent clusters $\left(\alpha^{+}\right.$and $\alpha^{-}$ being the positive and negative parts of $\left.\alpha: \alpha=\alpha^{+}-\alpha^{-}\right)$. We call (2.2) the excess decomposition of $\mathcal{K}$.

If $\xi: f=0$ is a curve on $S$ and $e_{u}(\xi)$ is the multiplicity of $\xi$ at $u$, we have the proximity equality:

$$
e_{p}(\xi)=\sum_{q \rightarrow p} e_{q}(\xi) \quad \text { for every } p \in \mathcal{N}_{O} .
$$

If $\pi_{K}: S_{K} \longrightarrow S$ is the composition of the blowing-ups of all the points in some cluster $K$ with an admissible order, write $\left\{E_{p}\right\}_{p \in K}$ for the irreducible components of the exceptional divisor of $\pi_{K}$ and

$$
\mathbf{A}=-P_{K}^{t} P_{K}
$$

for the associated intersection matrix of $\left\{E_{p}\right\}_{p \in K}$ (4.5.5 of [4]). For any $p \in K$, denote by $v_{p}(\xi)$ the value of $f$ relative to the divisorial valuation associated with $E_{p}$. If $v=\left(v_{p}\right)_{p \in K}$ and $e=\left(e_{p}\right)_{p \in K}$ are the vectors of values and multiplicities, respectively, we have

$$
v(\xi)=P_{K}^{-1} e(\xi) .
$$

We also write $v_{p}(I)=\min _{f \in I}\left\{v_{p}(f)\right\}$ and $\mathcal{E}_{p}=\sum_{q \in K} v_{q}\left(I_{p}\right) E_{q}$. We denote by $\mathbf{1}_{p}$ the $K$-vector having all its entries equal to 0 but the corresponding to $p$ which is 1 . Use $|\cdot|$ for the intersection number on $S_{K}$ and [, ] $O$ for intersection multiplicity at $O$. Write $\widetilde{\xi}^{K}$ for the strict transform of $\xi$ on $S_{K}$. We have the projection formula for $\pi_{K}:\left|\left(\pi_{K}\right)^{*}(\xi) \cdot D\right|_{S_{K}}=\left[\xi,\left(\pi_{K}\right)_{*}(D)\right]_{O}$ for any curve $D$ on $S_{K}$.

Sandwiched singularities. Take, once and for all, a complete $\mathfrak{m}$-primary ideal $I$ in $(R, \mathfrak{m})$ and write $\mathcal{K}=(K, \nu)$ for the cluster of base points of $I, \mathcal{K}=B P(I)$. Write $\pi: X=B l_{I}(S) \longrightarrow S$ for the blowing-up of $I$, that is, $X=\operatorname{Proj}\left(B_{I} R\right)$ where

$$
B_{I} R=R \oplus I \oplus I^{2} \oplus \ldots
$$


The singularities of $X$ are sandwiched and we have a commutative diagram

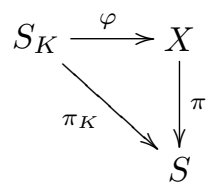

where the morphism $\varphi$, given by the universal property of the blowing-up, is the minimal resolution of the singularities of $X$ (Remark 1.4 of [17]). Recall that there is a bijection between the set of simple ideals $\left\{I_{p}\right\}_{p \in \mathcal{K}_{+}}$in the (Zariski) factorization of $I$ and the set of irreducible components of $\pi^{-1}(O)$ ([17] Corollary 1.5; see also [14] Proposition 21.3). We write $\left\{L_{p}\right\}_{p \in \mathcal{K}_{+}}$for the set of these components. For $p \in \mathcal{K}_{+}$, write

$$
\mathcal{L}_{p}=\sum_{q \in \mathcal{K}_{+}} v_{q}\left(I_{p}\right) L_{q}
$$

For every singularity $Q \in X$, write $T_{Q}=\left\{p \in K \mid \varphi_{*}\left(E_{p}\right)=Q\right\}$ so that $\left\{E_{p}\right\}_{p \in T_{Q}}$ is the set of the exceptional components of $S_{K}$ contracting to $Q$.

Every curve $C$ on $X$ can be written in a unique way as $C=\widetilde{\eta}+L$, where $\eta$ is a curve on $S$ and $L=\sum_{p \in \mathcal{K}_{+}} n_{p} L_{p}$ (with $n_{p} \in \mathbb{Z}_{\geq 0}$ ) is the exceptional part of $C$. Notice that $\eta$ is just the projection of $C$ by $\pi$ and that $\eta$ is empty if and only if $C$ has exceptional support. It is possible to associate to $C$ a $\mathbb{Q}$-Cartier exceptional divisor $D_{C}$ on $S_{K}$, defined by the condition

$$
\left|D_{C} \cdot E_{p}\right|_{S_{K}}=-\left|\widetilde{C}^{S_{K}} \cdot E_{p}\right|_{S_{K}} \quad \forall p \in K
$$

where $\widetilde{C}^{S_{K}}$ is the strict transform of $C$ on $S_{K}$ (see II (b) of [15]). On the other hand, if $D$ is a divisor on $S_{K}$ such that $\left|D \cdot E_{p}\right|=0$ for every $p \in K_{0}$, then $D$ is the total transform of some Cartier divisor on $X$ (see the proof of Theorem 4 of [2]). Because of this, we have

Lemma 2.1. $C$ is Cartier if and only if $D_{C}$ is a Weil divisor on $S_{K}$.

Proof. Since $S_{K}$ is a non-singular surface, if $D_{C}$ is a Weil divisor on $S_{K}$, then it is also a Cartier divisor, so is $\widetilde{C}^{S_{K}}+D_{C}$. The discussion above together with (2.7) shows that $\widetilde{C}^{S_{K}}+D_{C}$ is the total transform of some Cartier divisor on $X$. This Cartier divisor is necessarily $C$. The converse is immediate.

If $\xi$ is a curve on $S$, write $\widetilde{\xi}$ and $\xi^{*}$ for the strict and total transforms of $\xi$ on $X$, so that $\xi^{*}=\widetilde{\xi}+\mathcal{L}_{\xi}$ where $\mathcal{L}_{\xi}=\sum_{p \in \mathcal{K}_{+}} v_{p}(\xi) L_{p}$. Analogously, if $J \in \mathbf{I}$, we write $\mathcal{L}_{J}=\sum_{q \in \mathcal{K}_{+}} v_{q}(J) L_{q}$. If $\zeta$ is another curve on $S$ sharing no branches with $\xi$, from the projection formula for $\pi_{K}$ and (2.7), it is straightforward to show the projection formula for $\pi:[\xi, \zeta]_{O}=\left|\xi^{*} \cdot \widetilde{\zeta}\right|_{X}$ and

$$
\left|\xi^{*} \cdot L_{p}\right|_{X}=0 \quad \text { for every } p \in \mathcal{K}_{+} .
$$

Throughout this paper, we adopt the following convention: curves on $S$ are represented by greek letters, while curves on $X$ or $S_{K}$ are represented by capital latin letters. 


\section{Principality of curves on sandwiched surfaces}

First, we study the Picard group of $X$ and provide a criterium to check whether the strict transform on $X$ of a curve on $S$ is a Cartier divisor. Our first result states that the Picard group of $X$ is the free abelian group generated by the divisors $\left\{\mathcal{L}_{p}\right\}_{p \in \mathcal{K}_{+}}$ introduced in (2.6).

Theorem 3.1. $\operatorname{Pic}(X) \simeq \bigoplus_{p \in \mathcal{K}_{+}} \mathbb{Z} \mathcal{L}_{p}$.

In order to prove Theorem 3.1, we need the following lemma.

Lemma 3.2. (a) The set $\left\{\mathcal{E}_{p}\right\}_{p \in K}$ is a basis of the $\mathbb{Q}$-vector space generated by $\left\{E_{p}\right\}_{p \in K}$. The matrix of the change of basis from $\left\{\mathcal{E}_{p}\right\}_{p \in K}$ to $\left\{E_{p}\right\}_{p \in K}$ is $-\mathbf{A}$.

(b) The set $\left\{\mathcal{L}_{u}\right\}_{u \in \mathcal{K}_{+}}$is a basis of the $\mathbb{Q}$-vector space generated by $\left\{L_{u}\right\}_{u \in \mathcal{K}_{+}}$.

Proof. (a) Notice that the matrix $\mathbf{A}=-P_{K}^{t} P_{K}$ is negative definite since $P_{K}$ is unimodular. By applying (2.7) to a generic curve through $\mathcal{K}(p)$, we obtain that $\mathbf{A}\left(v_{q}\left(I_{p}\right)\right)_{q \in K}=-\mathbf{1}_{p}$. Thus $\mathbf{A} \mathcal{E}_{p}=-E_{p}$ and the claim follows.

(b) It is enough to show that the $\left\{\mathcal{L}_{u}\right\}_{u \in \mathcal{K}_{+}}$are linearly independent. Assume that there exist rational numbers $\left\{a_{u}\right\}_{u \in \mathcal{K}_{+}}$such that $\sum_{u \in \mathcal{K}_{+}} a_{u} \mathcal{L}_{u}=0$. By multiplying by an integer, we can assume that $a_{u} \in \mathbb{Z}$ for every $u \in \mathcal{K}_{+}$. Let $\gamma_{u}$ be a generic curve going through $\mathcal{K}(u)$ and missing all points after $u$ in $K$, and write

$$
\sum_{u \in \mathcal{K}_{+}} a_{u} \gamma_{u}=\zeta_{1}-\zeta_{2}
$$

where $\zeta_{1}=\sum_{u} a_{u}^{+} \gamma_{u}$ and $\zeta_{2}=\sum_{u} a_{u}^{-} \gamma_{u}$. Then, by assumption, we have $\mathcal{L}_{\zeta_{1}}=$ $\sum_{u} a_{u}^{+} \mathcal{L}_{u}$ and $\mathcal{L}_{\zeta_{2}}=\sum_{u} a_{u}^{-} \mathcal{L}_{u}$ are equal. By taking total transforms on $S_{K}$, we reach a contradiction with (a).

Proof of Theorem 3.1. It is enough to show that any effective Cartier divisor $C$ is linearly equivalent to some divisor in $\bigoplus_{p \in \mathcal{K}_{+}} \mathbb{Z} \mathcal{L}_{p}$. Let $C=\widetilde{\eta}+L$ be a curve on $X$. Then, we have

$$
\eta^{*}=C-\left(\mathcal{L}_{\eta}-L\right)
$$

so it is clear that $C$ is linearly equivalent to $\mathcal{L}_{\eta}-L$. According to Lemma 2.1, we have that $\mathcal{L}_{\eta}-L$ is Cartier if and only if its total transform on $S_{K}$

$$
\varphi^{*}\left(\mathcal{L}_{\eta}-L\right)=\sum_{q \in K} b_{q} E_{q} \quad \text { for some } b_{q} \in \mathbb{Q}
$$

is a Weil divisor, that is, $b_{q} \in \mathbb{Z}$ for every $q \in \mathcal{K}$. On the other hand, by (b) of Lemma 3.2 , we can write $\mathcal{L}_{\eta}-L=\sum_{u \in \mathcal{K}_{+}} a_{u} \mathcal{L}_{u}$, for some rational numbers $\left\{a_{u}\right\}$. Now, $\varphi^{*}\left(\mathcal{L}_{u}\right)=\mathcal{E}_{u}$ and so,

$$
\varphi^{*}\left(\mathcal{L}_{\eta}-L\right)=\sum_{u \in \mathcal{K}_{+}} a_{u} \mathcal{E}_{u}
$$

is the expression of $\varphi^{*}\left(\mathcal{L}_{\eta}-L\right)$ in the basis $\left\{\mathcal{E}_{p}\right\}_{p \in K}$. By (a) of Lemma 3.2, $\left(a_{u}\right)_{u \in K}=$ $-\mathbf{A}\left(b_{u}\right)_{u \in K}$. Since the matrix $\mathbf{A}$ is unimodular, we infer that $C$ is Cartier if and only if all the $a_{u}$ are integers. 
The proof of Theorem 3.1 has given further information which is worth keeping for future reference.

Corollary 3.3. The curve $C=\widetilde{\eta}+L$ is a Cartier divisor on $X$ if and only if $\mathcal{L}_{\eta}-L \in \bigoplus_{u \in \mathcal{K}_{+}} \mathbb{Z} \mathcal{L}_{u}$. In this case, if $\mathcal{L}_{\eta}-L=\sum_{p \in \mathcal{K}_{+}} a_{p} \mathcal{L}_{p}$, then $a_{p}=\left|C \cdot L_{p}\right|_{X}$ for every $p \in \mathcal{K}_{+}$.

Proof. The first claim follows trivially from (3.1). For the second, let $\gamma_{p}$ be a generic curve going through $\mathcal{K}(p)$. By using the projection formula (2.8) applied to $\gamma_{p}$, it is immediate to see that

$$
\left|\mathcal{L}_{p} \cdot L_{q}\right|_{X}=-\delta_{p, q} \quad \text { for } p, q \in \mathcal{K}_{+}
$$

where $\delta_{p, q}$ is the Kronecker delta. Now, if $C$ is Cartier and $\mathcal{L}_{\eta}-L=\sum_{p} a_{p} \mathcal{L}_{p}$, the projection formula (2.8) and (3.3) say that $a_{q}=\left|C \cdot L_{q}\right|_{X}$.

Corollary 3.4. Let $\eta$ be a curve on $S$. Then, $\tilde{\eta}$ is a Cartier divisor on $X$ if and only if there exists some curve $\eta^{\prime}$ on $S$ such that $\mathcal{L}_{\eta^{\prime}}=\mathcal{L}_{\eta}$ and $\widetilde{\eta^{\prime}}$ goes through no singularity of $X$. Moreover, if $\widetilde{\eta}$ goes through some singularity of $X$, say $Q$, then $v_{u}(\eta)>v_{u}\left(\eta^{\prime}\right)$ for all $u \in T_{Q}$.

To prove Corollary 3.4, we need the following lemma, which is stated separately for future reference. For each $p \in K$, we write $\theta_{p}^{\eta}=\sum_{q \rightarrow p, q \notin K} e_{q}(\eta)$.

Lemma 3.5. If $\xi$ is a curve on $S$, then $\left|\widetilde{\xi}^{K} \cdot E_{p}\right|_{S_{K}}=\theta_{p}^{\xi}$. In particular, if $\xi$ is a generic curve going through some cluster $\mathcal{T}=(K, \tau)$, then $\widetilde{\xi}$ goes through some singularity $Q \in X$ if and only if there exists some $p \in T_{Q}$ such that $\rho_{p}^{\mathcal{T}}>0$.

Proof. By the projection formula applied to $\pi_{K}$, we have

$$
\left|\widetilde{\xi}^{K} \cdot E_{p}\right|_{S_{K}}=-\mathbf{1}_{p}^{t} \mathbf{A} v(\xi)
$$

where $\mathbf{1}_{p}^{t}$ is the transpose of the vector $\mathbf{1}_{p}$ defined in page 3 . By $(2.4)$, we know that $\mathbf{A}=-P_{K}^{t} P_{K}$. From (2.5), we infer that $\left|\widetilde{\xi}^{K} \cdot E_{p}\right|_{S_{K}}=e_{p}(\xi)-\sum_{q \in K, q \rightarrow p} e_{q}(\xi)$ and we apply (2.3). The second claim follows easily from the fact that under the assumption above, we have $\theta_{p}^{\xi}=\rho_{p}^{\mathcal{T}}$ for every $p \in K$.

Proof of Corollary 3.4. From Corollary 3.3, we know that $\tilde{\eta}$ is Cartier if and only if $\mathcal{L}_{\eta}=\sum_{p \in \mathcal{K}_{+}} a_{p} \mathcal{L}_{p}$, where every $a_{p}=\left|\widetilde{\eta} \cdot L_{p}\right|_{X}$ is a non-negative integer. In this case, let $\eta^{\prime}$ be a generic curve going through the cluster $\sum_{p \in \mathcal{K}_{+}} a_{p} \mathcal{K}(p)$. Then, we have $\mathcal{L}_{\eta^{\prime}}=\mathcal{L}_{\eta}$ and by Lemma 3.5 , it is clear that $\widetilde{\eta^{\prime}}$ does not go through any singularity of $X$. The converse follows easily from Corollary 3.3, too. Now, let $\eta: g=0$ and $\eta^{\prime}: g^{\prime}=0$ be equations for $\eta$ and $\eta^{\prime}$, respectively. Since $\mathcal{L}_{\eta}=\mathcal{L}_{\eta^{\prime}}$, it follows that $\tilde{\eta}$ is defined locally near $Q$ by $g / g^{\prime}=0$. Since $\widetilde{\eta}$ goes through $Q$, we have $v_{u}\left(g / g^{\prime}\right)>0$ for every $u \in T_{Q}$. The claim follows. 
Divisor class group of sandwiched singularities. Our next goal is to determine the divisor class group (Weil divisors modulo linear equivalence) of an open affine set containing the singularities of $X$. From this, we will obtain the divisor class group of any sandwiched singularity. To this aim, let $a$ be a generic element of $I$. Then, we can write

$$
a=\prod_{p \in \mathcal{K}_{+}} a_{p}^{1} \ldots a_{p}^{\rho_{p}^{\mathcal{K}}}
$$

where each $a_{p}^{i}$ is a generic element of $I_{p}$. Write $V_{a}$ for the open affine set of $X$ defined by $a \neq 0$, that is, if $I=\left(f_{0}, \ldots, f_{n}\right)$, then

$$
V_{a}=\operatorname{Spec} R\left[f_{0} / a, \ldots, f_{n} / a\right] .
$$

Observe that from the genericity of $a$, it follows that $V_{a}$ contains all the singularities of $X$. Write $L_{p}^{\prime}$ for the restriction of $L_{p}$ to $V_{a}$ and $\mathbf{L}^{\prime}=\bigoplus_{p \in \mathcal{K}_{+}} \mathbb{Z} L_{p}^{\prime}$. Then we have

Proposition 3.6. $\mathrm{Cl}\left(V_{a}\right) \simeq \mathbf{L}^{\prime} / \bigoplus_{p \in \mathcal{K}_{+}} \mathbb{Z} \mathcal{L}_{p}^{\prime}$.

Proof. Write $\mathbf{L}=\bigoplus_{p \in \mathcal{K}_{+}} \mathbb{Z} L_{p}$ and $U=X \backslash \pi^{-1}(O)$. Then, the restriction $\mathrm{Cl}(X) \rightarrow$ $\mathrm{Cl}(U)$ induces an exact sequence

$$
0 \rightarrow \mathbf{L} \rightarrow \mathrm{Cl}(X) \rightarrow \mathrm{Cl}(U) \rightarrow 0
$$

the injection on the left as there are no principal exceptional divisors on $X$. By restriction to $V_{a}$, this induces another exact sequence

$$
\mathbf{L}^{\prime} \stackrel{\psi}{\rightarrow} \mathrm{Cl}\left(V_{a}\right) \rightarrow \mathrm{Cl}\left(U^{\prime}\right) \rightarrow 0
$$

where $U^{\prime}=V_{a} \backslash \pi^{-1}(O)$. On the other hand, the birational morphism $\pi$ induces an isomorphism $U \simeq S \backslash\{O\}$ and from this, we have $\mathrm{Cl}(U)=0$ as it equals the Picard group of the regular local ring $R$ (in particular, $\mathrm{Cl}(X) \simeq \mathbf{L}$ ). Since $U^{\prime} \subset U$, we infer that $\mathrm{Cl}\left(U^{\prime}\right)=0$. Now, the kernel of $\psi$ is just $\bigoplus_{p \in \mathcal{K}_{+}} \mathbb{Z} \mathcal{L}_{p}^{\prime}$, where $\mathcal{L}_{p}^{\prime}$ is the restriction of $\mathcal{L}_{p}$ to $V_{a}$. Indeed, since $V_{a}$ contains the singularities of $X$, if $\sum_{p} b_{p} L_{p}^{\prime}$ is principal on $V_{a}$, then $\sum_{p} b_{p} L_{p}$ must be locally principal on $X$ and by virtue of Theorem 3.1, we see that $\operatorname{Ker} \psi \subset \bigoplus_{p \in \mathcal{K}_{+}} \mathbb{Z} \mathcal{L}_{p}^{\prime}$. Of course, $\mathcal{L}_{p}^{\prime}$ is principal on $V_{a}$, defined by the equation $a_{p}=0$. The claim follows.

Example 3.7. (cf. Example 6.5.2 of [11]) Take $R=\mathbb{C}\{x, y\}$ and $I=\left(x, y^{2}\right) \in \mathbf{I}$. Let $X=B l_{I}(S)$ and take $V_{x}$ the open affine subset of $X$ defined by $x \neq 0$. Then, $V_{x}$ is just an affine quadric cone defined by the equation $z x-y^{2}=0$. The cluster $\mathcal{K}=B P(I)$ has two points: $p_{1}=O$ and $p_{2}$ in the direction of $x=0$ over the first neighbourhood of $p_{1}$. The Enriques diagram of $\mathcal{K}$ is shown in Figure 1 (Enriques diagrams are explained in [8] Book IV Chapter 1, and also in [4] §3.9). We have $\mathcal{L}_{p_{2}}=2 L_{p_{2}}$ and by virtue of Proposition 3.6, $\mathrm{Cl}\left(V_{x}\right) \simeq \mathbb{Z} / 2 \mathbb{Z}$ and it is generated by the germ of the exceptional divisor $L_{p_{2}}^{\prime}$. Finally notice that $L_{p_{2}}^{\prime}$ is just a ruling on $V_{x}$.

From an algebraic point of view, a sandwiched singularity $\mathcal{O}$ dominating $R$ is just a normal birational extension of the regular ring $R$ (see [12]). From the above results, the divisor class group of any sandwiched singularity can be determined. Let $I \in \mathbf{I}$ be an ideal such that $\mathcal{O}$ lies on the surface $X$ obtained by blowing up $I$. By Corollary 1.14 of [17], the ideal $I$ can be chosen so that $\mathcal{O}$ is the only singularity on $X$ (i.e. 

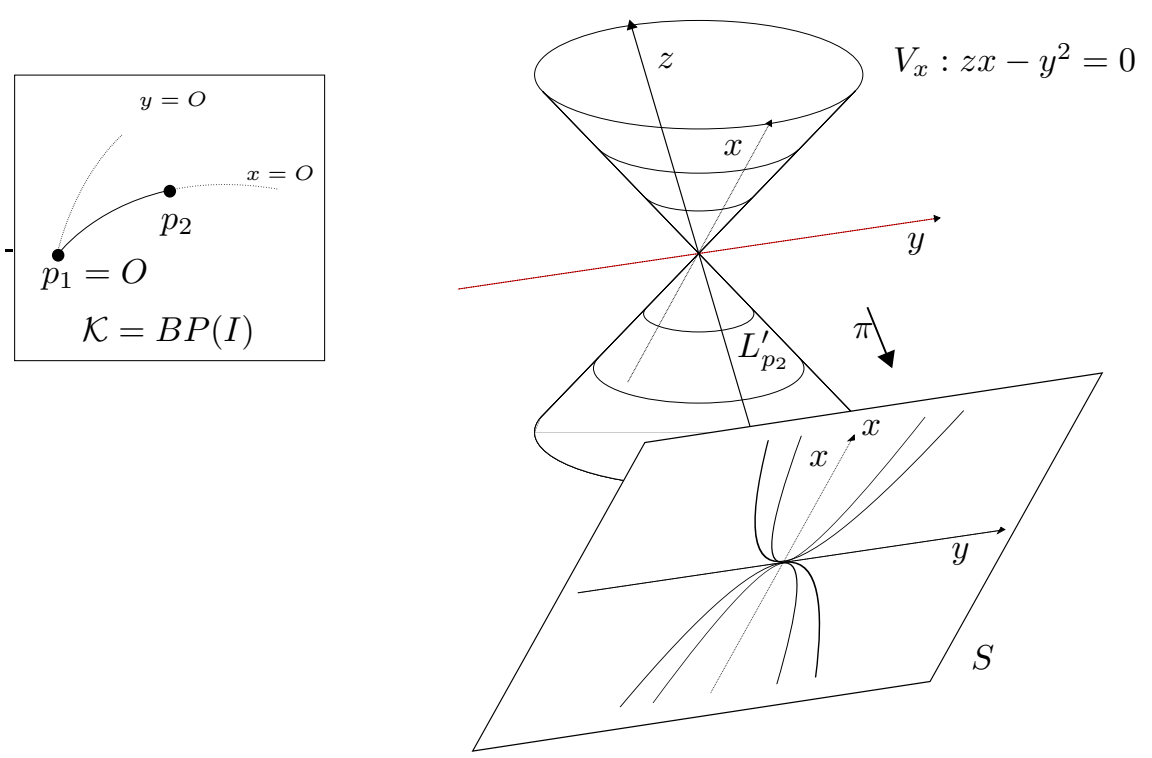

Figure 1. Birational projection of the affine quadric cone on a non-singular surface $S$ in Example 3.7. In the square, the Enriques diagram of $\mathcal{K}$ is shown.

there is only one singular point $Q \in X$ and $\mathcal{O} \simeq \mathcal{O}_{X, Q}$ and the strict transforms of the exceptional components $\left\{L_{p}\right\}_{p \in \mathcal{K}_{+}}$on the minimal resolution of $(X, Q)$ have self-intersection -1). As above, write $\mathcal{K}=B P(I)$ and $\mathcal{K}_{+}$for the dicritical points of $\mathcal{K}$. With the notation already introduced, Proposition 3.6 implies that

Corollary 3.8. $\mathrm{Cl}\left(\mathcal{O}_{X, Q}\right) \simeq \mathbf{L}^{\prime} / \bigoplus_{p \in \mathcal{K}_{+}} \mathbb{Z} \mathcal{L}_{p}^{\prime}$

Remark 3.9. Recall that the only normal birational extensions of $R$ being factorial are those which are already regular ([12]; Theorems 20.1 and 25.1 of [14]). In particular, we have

$$
\mathbf{L}^{\prime}=\bigoplus_{p \in \mathcal{K}_{+}} \mathbb{Z} \mathcal{L}_{p}^{\prime} \quad \Leftrightarrow \quad \operatorname{Cl}\left(\mathcal{O}_{X, Q}\right)=0 \quad \Leftrightarrow \quad \mathcal{O}_{X, Q} \text { is regular. }
$$

Example 3.10. (Divisor class group of primitive singularities) Primitive singularities are those singularities that can be obtained by blowing up a simple complete ideal (Definition I.3.1 of [17]). Let $I_{p}$ be a simple ideal where $p$ is a free point and let $X=$ $B l_{I_{p}}(S)$ be the surface obtained by blowing-up $I_{p}$. Write $Q$ for the only singularity in $X$. Then, Corollary 3.8 gives that

$$
\mathrm{Cl}\left(\mathcal{O}_{X, Q}\right) \simeq \mathbb{Z} / \mathcal{K}(p)^{2} \mathbb{Z}
$$

where $\mathcal{K}(p)^{2}$ is the intersection multiplicity at $O$ of two curves defined by generic elements of $I_{p}$. 


\section{Unloading relative to a curve}

Given a curve $\eta: f=0$ on $S$, this section is devoted to introducing a variation of the unloading procedure for clusters which takes into account the points and multiplicities of $\eta$ (cf. 4.6 of [4]). This process will be used in the next section to check whether a given curve on the sandwiched surface $X$ is principal near the singularities.

Let $\mathcal{P}$ be a weighted cluster whose points are contained in $K$, so that by adding points with virtual multiplicity zero (if necessary) we can write $\mathcal{P}=(K, \varrho)$. Write $\bar{v}(\mathcal{P})$ for the vector of virtual values of $\mathcal{P}$, that is (see $(2.5)$ )

$$
\bar{v}(\mathcal{P})=P_{K}^{-1} \varrho .
$$

Notice that $\mathcal{P}$ is consistent if and only if $\bar{v}(\mathcal{P})=v\left(H_{\mathcal{P}}\right)$. We associate to $\mathcal{P}$ a sheaf of ideals on $X$ by taking

$$
\mathcal{H}_{\mathcal{P}}=\left\{g \in B_{I} R \mid v(g) \geq \bar{v}(\mathcal{P})\right\} .
$$

It is worth noting that if the base points of some ideal $J \in \mathbf{I}$ are contained in $K$, then

$$
J \mathcal{O}_{X}=\left\{g \in B_{I} R \mid v(g) \geq v(J)\right\}=\mathcal{H}_{B P(J)} .
$$

Next, we introduce two variations of the notion of consistency of clusters:

1. A cluster $\mathcal{P}=(K, \varrho)$ is $K_{0}$-consistent if $\rho_{q}^{\mathcal{P}} \geq 0$ for every point $q \in K_{0}$. We have

Lemma 4.1. If $\mathcal{P}$ is $K_{0^{-}}$consistent, then $\mathcal{H}_{\mathcal{P}}=H_{\mathcal{P}^{+}} \mathcal{O}_{X}\left(\mathcal{L}_{\mathcal{P}^{-}}\right)$.

Proof. Take the excess decomposition of $\mathcal{P}$ as in (2.2): $\mathcal{P}=\mathcal{P}^{+}-\mathcal{P}^{-}$. Since both $\mathcal{P}^{+}$and $\mathcal{P}^{-}$are consistent, we have $\bar{v}(\mathcal{P})=v\left(H_{\mathcal{P}^{+}}\right)-v\left(H_{\mathcal{P}^{-}}\right)$. Then,

$$
\begin{aligned}
\mathcal{H}_{\mathcal{P}} \mathcal{O}_{X}\left(-\mathcal{L}_{\mathcal{P}^{-}}\right) & =\left\{g \in B_{I} R \mid v(g) \geq \bar{v}(\mathcal{P})+v\left(H_{\mathcal{P}^{-}}\right)\right\}= \\
& =\left\{g \in B_{I} R \mid v(g) \geq v\left(H_{\mathcal{P}^{+}}\right)\right\}=H_{\mathcal{P}^{+}} \mathcal{O}_{X}
\end{aligned}
$$

where the last equality follows from (4.1). From this, the claim follows.

2. We say that $\mathcal{T}=(K, \tau)$ is $\left(K_{0}, \eta\right)$-consistent if $\rho_{p}^{\mathcal{T}} \geq \theta_{p}^{\eta}$ for every point $p \in K_{0}$.

In what follows, we describe what we call an $\eta$-unloading step: it applies to any $\left(K_{0}, \eta\right)$-nonconsistent cluster $\mathcal{T}$ to give a $\left(K_{0}, \eta\right)$-consistent cluster $\widetilde{\mathcal{T}}$ with $(f) \cap \mathcal{H}_{\mathcal{T}}=$ $(f) \cap \mathcal{H}_{\widetilde{\mathcal{T}}}$

$\eta$-unloading. Assume that there is some point $p \in K_{0}$ such that $\rho_{p}^{\mathcal{T}}<\theta_{p}^{\eta}$. Define $n$ as the least integer greater than or equal to $\left(\theta_{p}^{\eta}-\rho_{p}^{\mathcal{T}}\right) /\left(m_{p}+1\right)$, where $m_{p}$ is the number of points in $K$ proximate to $p$. In this case, $\eta$-unloading the multiplicity on $p$ consists of defining a new cluster $\mathcal{T}^{\prime}=\left(K, \tau^{\prime}\right)$ by the rules

$$
\begin{aligned}
\tau_{p}^{\prime} & =\tau_{p}+n \\
\tau_{q}^{\prime} & =\tau_{q}-n \text { if } q \rightarrow p \\
\tau_{q}^{\prime} & =\tau_{q} \text { otherwise. }
\end{aligned}
$$

Notice that usual unloading is recovered by taking $\eta$ equal to the empty germ. 


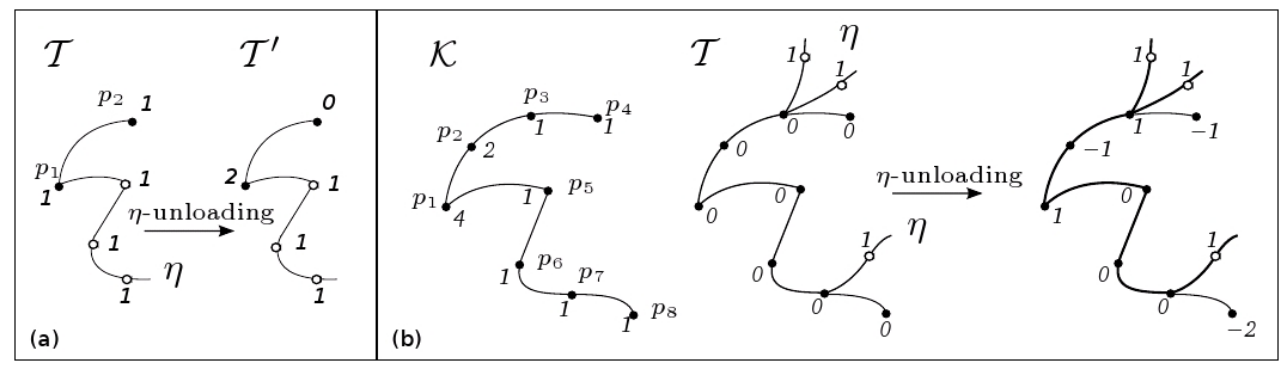

Figure 2. Enriques diagrams illustrating the $\eta$-unloading procedure. White dots represent points of the curve $\eta$ which are not in $K$. (a) The Enriques diagrams of $\mathcal{T}$ and $\mathcal{T}^{\prime}$ in Example 4.2. (b) The Enriques diagrams of $\mathcal{K}, \mathcal{T}$ and the cluster obtained from $\mathcal{T}$ by performing $\eta$-unloading on $K_{0}$ in Example 4.5.

Example 4.2. Take $\eta: y^{2}-x^{3}=0$ in Example 3.7. Write $\mathcal{T}=(K, \tau)$ with $\tau_{p_{1}}=\tau_{p_{2}}=1$. Figure 2(a) shows the Enriques diagram of $\mathcal{T}$. The cluster $\mathcal{T}$ fails to be $\left(K_{0}, \eta\right)$-consistent because the excess at $p_{1}$ is -2 . By applying $\eta$-unloading on $p_{1}$ we obtain the cluster $\mathcal{T}^{\prime}$, which is $\left(K_{0}, \eta\right)$-consistent.

Observe that in general, $\mathcal{H}_{\mathcal{T}} \subsetneq \subsetneq \mathcal{H}_{\mathcal{T}}$. However, we have

Lemma 4.3. $(f) \cap \mathcal{H}_{\mathcal{T}}=(f) \cap \mathcal{H}_{\mathcal{T}^{\prime}}$.

Proof. Write $\bar{v}$ and $\bar{v}^{\prime}=\bar{v}+n \mathbf{1}_{p}$ for the vector of virtual values of $\mathcal{T}$ and $\mathcal{T}^{\prime}$, respectively. By definition, we have $\mathcal{H}_{\mathcal{T}}=\left\{g \in B_{I} R \mid v(g) \geq \bar{v}\right\}$ and $\mathcal{H}_{\mathcal{T}^{\prime}}=\{g \in$ $\left.B_{I} R \mid v(g) \geq \bar{v}^{\prime}\right\}$. Clearly, $\mathcal{H}_{\mathcal{T}^{\prime}} \subset \mathcal{H}_{\mathcal{T}}$ and $(f) \cap \mathcal{H}_{\mathcal{T}}, \subset(f) \cap \mathcal{H}_{\mathcal{T}}$. To prove the other inclusion, let $g \in(f) \cap \mathcal{H}_{\mathcal{T}}$. Then, $g=f h$ for some $h \in B_{I} R$ and we must show that $g \in \mathcal{H}_{\mathcal{T}^{\prime}}$, i.e. that $v(g) \geq \bar{v}^{\prime}$. Write $\mathbf{A}=\left(\alpha_{p, q}\right)_{p, q \in K}$ for the intersection matrix of $\left\{E_{p}\right\}_{p \in K}$. Then, from (2.4) and (2.5) it is straightforward to show that

$$
\sum_{q \in K} v_{q}(f) \alpha_{p, q}=-\theta_{p}^{\eta} \quad \text { for every } p \in K .
$$

On the other hand, from the equality $\varphi^{*}(h)=\widetilde{h}^{K}+\sum_{q \in K} v_{q}(h) E_{q}$ and the fact that $\left|\varphi^{*}(h) \cdot E_{p}\right|_{S_{K}}=0$, we have

$$
\sum_{q \in K} v_{q}(h) \alpha_{p, q}=\left|\left(\varphi^{*}(h)-\widetilde{h}^{K}\right) \cdot E_{p}\right|_{S_{K}} \leq 0 .
$$

From (4.2) and (4.3), it follows that $\sum_{q \in K} v_{q}(g) \alpha_{p, q} \leq-\theta_{p}^{\eta}$. Since $g \in \mathcal{H}_{\mathcal{T}}$, we have $\sum_{q \neq p} v_{q}(g) \alpha_{p, q} \geq \sum_{q \neq p} \bar{v}_{q} \alpha_{p, q}$. From these inequalities, we obtain

$$
\left(v_{p}(g)-\bar{v}_{p}\right) \alpha_{p, p} \leq-\theta_{p}^{\eta}-\sum_{q \in K} v_{q} \alpha_{p, q}=-\theta_{p}^{\eta}+\rho_{p}^{\mathcal{T}} .
$$

Since $\alpha_{p, p}=-m_{p}-1$, it follows that $v_{p}(g)-\bar{v}_{p} \geq\left(\theta_{p}^{\eta}-\rho_{p}^{\mathcal{T}}\right) /\left(m_{p}+1\right)$. Now, the definition of $n$ gives that $v_{p}(g)-\bar{v}_{p} \geq n$ and hence, $v_{p}(g) \geq \bar{v}_{p}+n=\bar{v}_{p}^{\prime}$.

The following proposition shows that after a finite sequence of $\eta$-unloading steps as above a $\left(K_{0}, \eta\right)$-consistent cluster is obtained. Notice that this happens no matter 
which point of $K_{0}$ is chosen to unload at each step. Hence, the procedure does not need a special strategy.

Proposition 4.4. Assume that $\mathcal{T}$ is not $\left(K_{0}, \eta\right)$-consistent. Put $\mathcal{T}_{0}=\mathcal{T}$ and as long as $\mathcal{T}_{i-1}$ is not $\left(K_{0}, \eta\right)$-consistent, define $\mathcal{T}_{i}$ from $\mathcal{T}_{i-1}$ by $\eta$-unloading on a suitable point of $K_{0}$. Then, there is some $m$ such that $\mathcal{T}_{m}$ is $\left(K_{0}, \eta\right)$-consistent and $(f) \cap \mathcal{H}_{\mathcal{T}}=$ $(f) \cap \mathcal{H}_{\mathcal{T}_{m}}$.

Proof. Write $v^{i}$ for the system of virtual values of $\mathcal{T}_{i}$. Then, we know that $v^{i}>v^{i-1}$. On the other hand, take any $g \in(f) \cap \mathcal{H}_{\mathcal{T}}$. By using induction and Lemma 4.3, we have $g \in(f) \cap \mathcal{H}_{\mathcal{T}_{i}}$ for any $i$, so $v(g) \geq v^{i}$. Therefore, the increasing sequence of values $v^{i}$ must be finite as they are bounded above by $v(g)$. Since $(K, \eta)$-unloading can be applied as long as there is some point $q \in K_{0}$ with $\rho_{q}^{\mathcal{T}}<\theta_{q}^{\eta}$, this shows that after a finite number of steps, we reach some cluster $\mathcal{T}_{m}$ such that $\rho_{q}^{\mathcal{T}} \geq \theta_{q}^{\eta}$ for all $q \in K_{0}$.

Example 4.5. Take a cluster $\mathcal{K}$ with proximities and multiplicities as in the Enriques diagram on the left of Figure 2(b). The dicritical points of $\mathcal{K}$ are $p_{2}, p_{4}$ and $p_{8}$. Take a curve $\eta$ composed of three branches: two generic branches through $p_{3}$ and one generic branch $p_{7}$. Define $\mathcal{T}=(K, \tau)$ with $\tau_{p}=0$ for every $p \in K$. The cluster obtained by performing $\eta$-unloading on $\mathcal{T}$ is shown on the right of Figure 2(b).

\section{Local principality of curves on sandwiched singularities}

Let $C$ be an effective Weil divisor on $X$. Write as above $C=\widetilde{\eta}+L$ where $\eta: f=0$ is a curve on $S$ (which may be empty) and $L=\sum_{p \in \mathcal{K}_{+}} n_{p} L_{p}$, where $\left\{n_{p}\right\}_{p \in K_{+}}$are non-negative integers. From the procedure introduced in the preceding section, we will obtain a $K_{0}$-consistent cluster $\mathcal{R}_{C}$ and a curve $\xi_{C}$ on $S$. While the excesses of $\mathcal{R}_{C}$ will provide information about the local principality of $C$ on $X$ (Theorem 5.3), the multiplicities of $\widetilde{\xi_{C}}$ will give the minimal number of generators of the ideal of $C$ at the singular points of $X$ (Proposition 5.6).

To this aim, we begin by defining an auxiliary cluster $\mathcal{T}_{C}$ : write $\mathbf{n}=\left(n_{p}\right)_{p \in K}$ with $n_{p}=0$ if $p \notin \mathcal{K}_{+}$and take

$$
\mathcal{T}_{C}=(K, \tau) \quad \text { with } \tau=P_{K} \mathbf{n}
$$

i.e., the multiplicities of $\mathcal{T}_{C}$ are taken so that $\bar{v}\left(\mathcal{T}_{C}\right)=\mathbf{n}$. By virtue of Proposition 4.4, after finitely many $\eta$-unloading steps on $\mathcal{T}_{C}$, we reach a cluster $\widetilde{\mathcal{T}_{C}}=(K, \widetilde{\tau})$ such that:

1. $\rho_{p}^{\widetilde{\tau_{C}}} \geq \theta_{p}^{\eta}$ for every $p \in K_{0}$

2. $(f) \cap \mathcal{H}_{\mathcal{T}_{C}}=(f) \cap \mathcal{H}_{\widetilde{\mathcal{T}_{C}}}$.

Moreover, since no unloading is performed on $\mathcal{K}_{+}$, we also have

3. $\bar{v}_{p}\left(\widetilde{\mathcal{T}_{C}}\right)=n_{p}$ for each $p \in \mathcal{K}_{+}$.

Now, define the cluster $\mathcal{R}_{C}$ by taking

$$
\mathcal{R}_{C}=(K, r) \quad \text { with } r=\widetilde{\tau}-e(\eta) .
$$

Lemma 5.1. (a) $\mathcal{R}_{C}$ is $K_{0}$-consistent;

(b) $(f) \cap \mathcal{H}_{\mathcal{T}_{C}}=f \mathcal{H}_{\mathcal{R}_{C}}$. 
ProOF. (a) If $p \in K_{0},(2.3)$ and the definition of $\widetilde{\mathcal{T}_{C}}$ imply that $\rho_{p}^{\mathcal{R}_{C}}=\widetilde{\rho_{p}}-\theta_{p}^{\eta} \geq 0$.

(b) Assume that $g=f u \in \mathcal{H}_{\mathcal{T}_{C}}$. We know that $(f) \cap \mathcal{H}_{\mathcal{T}_{C}}=(f) \cap \mathcal{H}_{\widetilde{\mathcal{T}_{C}}}$ and so, $g \in \mathcal{H}_{\widetilde{\mathcal{T}_{C}}}$. Thus we have $v(g) \geq \bar{v}\left(\widetilde{\mathcal{T}_{C}}\right)=v(\eta)+\bar{v}\left(\mathcal{R}_{C}\right)$. Since $v(g)=v(\eta)+v(u)$, it follows that $v(u) \geq \bar{v}\left(\mathcal{R}_{C}\right)$ and $u \in \mathcal{H}_{\mathcal{R}_{C}}$. For the converse, assume that $g=f u$ with $u \in \mathcal{H}_{\mathcal{R}_{C}}$. Then, $v(u) \geq \bar{v}\left(\mathcal{R}_{C}\right)$ and $v(g) \geq \bar{v}\left(\widetilde{\mathcal{T}_{C}}\right)$. Now, $g \in(f) \cap \mathcal{H}_{\widetilde{\mathcal{T}_{C}}}=(f) \cap \mathcal{H}_{\mathcal{T}_{C}}$.

Proposition 5.2. The sheaf $f \mathcal{H}_{\mathcal{R}_{C}}$ is the sheaf of ideals of $C$.

ProOF. According to (b) of Lemma 5.1, we have $f \mathcal{H}_{\mathcal{R}_{C}}=(f) \cap \mathcal{H}_{\widetilde{\mathcal{T}_{C}}}$. Now, the sheaf of ideals of the strict transform of $C$ on $X$ is $(f) \cap B_{I} R=\bigoplus_{n \geq 0}(f) \cap I^{n}$. Hence, the sheaf $(f) \cap \mathcal{H}_{\mathcal{T}_{C}}$ is locally given by the sections $g$ containing $\tilde{\eta}$ and such that $v_{u}(g) \geq n_{u}$ for $u \in \mathcal{K}_{+}$. This is just the sheaf of ideals of $C$.

Notice that although $\mathcal{R}_{C}$ may not be consistent, by virtue of (a) in Lemma 5.1 the negative excesses (if any) appear only at points of $\mathcal{K}_{+}$. Thus, we can write (see (2.2))

$$
\mathcal{R}_{C}=\mathcal{R}_{C}^{+}-\mathcal{R}_{C}^{-}
$$

where both $\mathcal{R}_{C}^{+}$and $\mathcal{R}_{C}^{-}$are consistent clusters and the only dicritical points of $\mathcal{R}_{C}^{-}$ are in $\mathcal{K}_{+}$. We now show that from the excesses of $\mathcal{R}_{C}$ it is possible to deduce whether the curve $C$ is locally principal near any singularity $Q \in X$.

Theorem 5.3. Let $Q$ be a singularity of $X$. The following are equivalent:

1. $C$ is principal near $Q$;

2. the excess of $\mathcal{R}_{C}$ at any point of $T_{Q}$ is zero;

3. if $\xi_{C}$ is a generic curve going through $\mathcal{R}_{C}^{+}$, then $\widetilde{\xi}_{C}$ does not go through $Q$.

Proof. Let $\xi_{C}$ and $\zeta_{C}$ be generic curves going through $\mathcal{R}_{C}^{+}$and $\mathcal{R}_{C}^{-}$, respectively. First of all, we claim that $C+\widetilde{\xi}_{C}$ is a Cartier divisor on $X$. To show this, recall that $n_{p}=\bar{v}_{p}\left(\widetilde{T_{C}}\right)=\bar{v}_{p}\left(\mathcal{R}_{C}\right)+v_{p}(\eta)$. Write $\mathcal{L}_{\mathcal{R}_{C}}=\sum_{q \in \mathcal{K}_{+}} \bar{v}_{q}\left(\mathcal{R}_{C}\right) L_{q}$, so that $L=\mathcal{L}_{\eta}+\mathcal{L}_{\mathcal{R}_{C}}$. Now, we can write

$$
\left.C=\widetilde{\eta}+\mathcal{L}_{\eta}+\mathcal{L}_{\mathcal{R}_{C}}=\left(\eta^{*}+\xi_{C}^{*}-\zeta_{C}^{*}\right)+\widetilde{\left(\widetilde{\zeta_{C}}\right.}-\widetilde{\xi_{C}}\right),
$$

and locally near $Q$, we have the following equality of germs

$$
C+\widetilde{\xi_{C}}=\eta^{*}+\xi_{C}^{*}-\zeta_{C}^{*}
$$

because $\widetilde{\zeta_{C}}$ goes through no singularity of $X$ (see 3.5 ). This proves the claim. Now, we prove the equivalence between 1. and 3. From (5.2), we know that $C$ is principal near $Q$ if and only if $\widetilde{\xi_{C}}$ is. This shows that if $\widetilde{\xi_{C}}$ goes not through $Q$, then $C$ is principal near $Q$. For the converse, assume that $C$ is locally principal near $Q$ and that the germ of $\widetilde{\xi_{C}}$ at $Q$ is not empty. The idea is to construct a new curve $\xi_{C}^{\prime}$ going through $\mathcal{R}_{C}^{+}$such that its strict transform on $X$ does not go through $Q$. From this, we will obtain a contradiction. Let $w h=0$ be an equation for $\xi_{C}$ on $S$, where $\gamma: h=0$ is composed of the branches of $\xi_{C}$ whose strict transforms on $X$ go through $Q$ and $w=0$ is composed of the remaining branches (if any). By construction, the germ of the strict transform of $\gamma$ at $Q$ equals the germ of $\widetilde{\xi_{C}}$, and so, $\widetilde{\gamma}$ is a Cartier divisor. 
By Corollary 3.4, there is a curve $\gamma^{\prime}: h^{\prime}=0$ on $S$ whose strict transform on $X$ goes through no singularity of $X$ and $v_{u}\left(h^{\prime}\right)=v_{u}(h)$ if $u \in \mathcal{K}_{+}$while

$$
v_{u}\left(h^{\prime}\right)<v_{u}(h) \quad \text { if } u \in T_{Q} .
$$

Take the curve

$$
\xi_{C}^{\prime}: w h^{\prime}=0 .
$$

Then $\mathcal{L}_{\xi_{C}^{\prime}}=\mathcal{L}_{\xi_{C}}$ and $\eta^{*}+\left(\xi_{C}^{\prime}\right)^{*}-\mathcal{L}_{\zeta_{C}}$ is a Cartier divisor on $X$ containing $C$. According to Proposition 5.2, it follows that if $g=0$ is an equation for $\zeta_{C}$, then $f\left(w h^{\prime} / g\right) \in f \mathcal{H}_{\mathcal{R}_{C}}$, so $w h^{\prime} / g \in \mathcal{H}_{\mathcal{R}_{C}}$. From this, $w h^{\prime} \in H_{\mathcal{R}_{C}^{+}}$and $v_{u}\left(w h^{\prime}\right) \geq v_{u}\left(H_{\mathcal{R}_{C}^{+}}\right)$. On the other hand, if $u \in T_{Q}$, an easy computation shows that $v_{u}\left(w h^{\prime}\right)=v_{u}\left(H_{\mathcal{R}_{C}^{+}}\right)-$ $v_{u}(h)+v_{u}\left(h^{\prime}\right)$ and so, $v_{u}(h) \leq v_{u}\left(h^{\prime}\right)$ which contradicts (5.3).

Now, to prove the equivalence between 2 . and 3., notice that by virtue of Lemma 3.5 , we have that $\widetilde{\xi_{C}}$ goes through $Q$ if and only if $\mathcal{R}_{C}^{+}$has positive excess at some point of $T_{Q}$. The claim follows from the fact that the excesses of $\mathcal{R}_{C}$ and $\mathcal{R}_{C}^{+}$are the same at the points of $T_{Q}$.

In practice, the easiest way of checking whether a curve $C$ is principal near some singularity $Q$ of $X$ is to use the Enriques diagrams to $\eta$-unload the multiplicities of the cluster $\mathcal{T}_{C}$ (see Figure 2). Then, substract the multiplicities of $\eta$ from $\widetilde{\mathcal{T}_{C}}$ to obtain $\mathcal{R}_{C}$. By virtue of Theorem 5.3, $C$ is locally principal near $Q$ if and only if the excess of $\mathcal{R}_{C}$ at every point of $T_{Q}$ is zero. We illustrate this with two examples.

Example 5.4. In Example 3.7, the strict transform of $\eta: y^{2}-x^{3}=0$ on $X$ goes through the singularity and it is locally principal. Indeed, if we take $C=\widetilde{\eta}$, then $\mathcal{R}_{C}^{+}$ is empty and $\mathcal{R}_{C}^{-}=\mathcal{K}$.

Example 5.5. Take $I \in \mathbf{I}$ with cluster of base points $\mathcal{K}$ as in Example 4.5. The surface $X=B l_{I}(S)$ has three exceptional components: $L_{p_{2}}, L_{p_{4}}$ and $L_{p_{8}}$, and two singularities: $Q_{1}$ with $T_{Q_{1}}=\left\{p_{3}\right\}$ and $Q_{2}$ with $T_{Q_{2}}=\left\{p_{1}, p_{5}, p_{6}, p_{7}\right\}$. Define $C_{1}=\widetilde{\eta}$. The Enriques diagram on the center and the right of Figure 2(b) shows the multiplicities of $\mathcal{T}_{C_{1}}$ and $\widetilde{\mathcal{T}_{C_{2}}}$, respectively. The Enriques diagrams of $\mathcal{R}_{C_{1}}$ is shown on Figure 3 . We see that the excess of $\mathcal{R}_{C_{1}}$ at $p_{3}$ is zero, while the excesses at $p_{1}, p_{5}, p_{6}, p_{4}$ are $2,0,0,1$, respectively. It follows that $C_{1}$ is locally principal at $Q_{1}$, but not at $Q_{2}$ (see Theorem 5.3). Now, take the curve $C_{2}=\widetilde{\eta}+2 L_{p_{2}}+L_{p_{8}}$. In this case, the multiplicities for $\mathcal{T}_{C_{2}}$ are taken as $\tau_{p_{2}}=2, \tau_{p_{4}}=-2, \tau_{p_{8}}=1$ and $\tau_{p}=0$ if $p \in K_{0}$. After performing $\eta$-unloading on the points of $K_{0}$ and substracting the multiplicities of $\eta$, we obtain $\mathcal{R}_{C_{2}}$ as on the right of Figure 3. Since there are no dicritical points of $\mathcal{R}_{C_{2}}$ on $T_{Q_{1}}$ nor $T_{Q_{2}}$, the curve $C_{2}$ is a Cartier divisor.

Local systems of generators for the ideals of curves. As a consequence of the results proved above, we determine the minimal number of generators of the ideal of a curve $C$ in the local ring of any singularity of $X$. Keeping the notations as above, we have seen in Lemma 4.1 that

$$
\mathcal{H}_{\mathcal{R}_{C}}=H_{\mathcal{R}_{C}^{+}} \mathcal{O}_{X}\left(\mathcal{L}_{\mathcal{R}_{C}^{-}}\right) .
$$




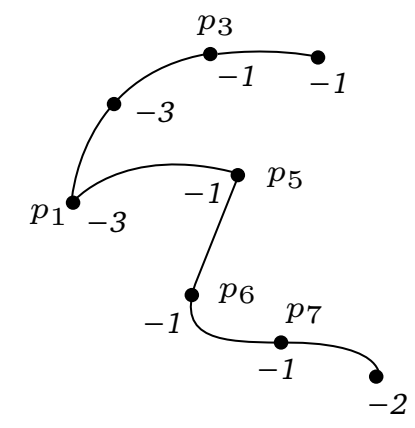

$\mathcal{R}_{C_{1}}$

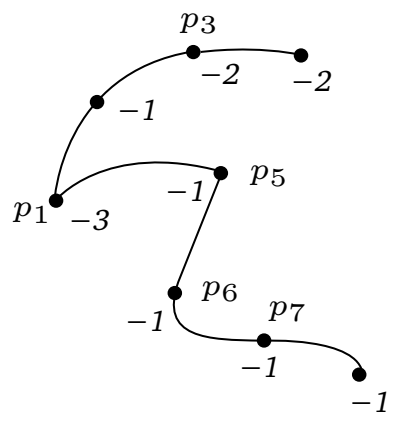

$\mathcal{R}_{C_{2}}$

Figure 3. The Enriques diagrams of $\mathcal{R}_{C_{1}}$ and $\mathcal{R}_{C_{2}}$ in Example 5.5.

Proposition 5.6. The germ of $C$ at $Q$ can be defined by $1+\operatorname{mult}_{Q}\left(\widetilde{\xi_{C}}\right)$ local equations. Moreover, this is the minimal number of generators of the ideal of $C$ at $Q$.

Proof. First of all, write $I_{Q}=\Gamma\left(X, \mathcal{M}_{Q} I \mathcal{O}_{X}\right) \in \mathbf{I}$, where $\mathcal{M}_{Q}$ is the sheaf of ideals of $Q$. By Theorem 3.5 of [9], we know $I_{Q}$ has codimension one in $I$. We have that $I \mathcal{O}_{X}=\mathcal{O}_{X}\left(-\mathcal{L}_{I}\right)$ and by Theorem 3.5 of $[9], I_{Q} \mathcal{O}_{X}=\mathcal{M}_{Q} \mathcal{O}_{X}\left(-\mathcal{L}_{I}\right)$. From this and Proposition 5.2, it follows that if $\mathcal{J}_{C}$ denotes the sheaf of ideals of $C$ on $X$, then $\mathcal{J}_{C}=(f I) \mathcal{H}_{\mathcal{R}_{C}}\left(\mathcal{L}_{I}\right)$ and $\mathcal{J}_{C} \mathcal{M}_{Q}=\left(f I_{Q}\right) \mathcal{H}_{\mathcal{R}_{C}}\left(\mathcal{L}_{I}\right)$. Now, it is straightforward to see that the minimal number of generators of the stalk of $\mathcal{J}_{C}$ at $Q$ is given by

$$
\begin{aligned}
\operatorname{dim}_{\mathbb{C}}\left(\frac{\mathcal{J}_{C, Q}}{\mathcal{M}_{Q} \mathcal{J}_{C, Q}}\right) & =\operatorname{dim}_{\mathbb{C}}\left(\frac{I}{I_{Q}}\right)+\left[H_{\mathcal{R}_{C}^{+}}, I_{Q}\right]_{O}-\left[H_{\mathcal{R}_{C}^{+}}, I\right]_{O}= \\
& =1+\left[H_{\mathcal{R}_{C}^{+}}, I_{Q}\right]_{O}-\left[H_{\mathcal{R}_{C}^{+}}, I\right]_{O}
\end{aligned}
$$

where $\left[J_{1}, J_{2}\right]_{O}$ means the intersection multiplicity of curves defined by generic elements of $J_{1}$ and $J_{2}$. By applying the projection formula for $\pi$, we have $\left[H_{\mathcal{R}_{C}^{+}}, I_{Q}\right]_{O}=$ $\left|\widetilde{\xi_{C}} \cdot\left(\widetilde{\zeta_{I_{Q}}}+\mathcal{L}_{I}\right)\right|_{X}$ and similarly, $\left[H_{\mathcal{R}_{C}^{+}}, I\right]_{O}=\left|\widetilde{\xi_{C}} \cdot\left(\widetilde{\zeta_{I}}+\mathcal{L}_{I}\right)\right|_{X}$, where $\zeta_{I_{Q}}$ and $\zeta_{I}$ are curves defined by generic elements of $I_{Q}$ and $I$, respectively. By the genericity of $\zeta_{I}$, we have $\left|\widetilde{\xi_{C}} \cdot \widetilde{\zeta_{I}}\right|_{X}=0$. The claim follows from the fact that $\widetilde{\zeta_{I_{Q}}}$ is a generic hypersurface section through $(X, Q)$ so that $\left[H_{\mathcal{R}_{C}^{+}}, I_{Q}\right]_{O}-\left[H_{\mathcal{R}_{C}^{+}}, I\right]_{O}=\left|\widetilde{\xi_{C}} \cdot \widetilde{\zeta_{I_{Q}}}\right|_{X}=\operatorname{mult}_{Q}\left(\widetilde{\xi_{C}}\right)$.

Observe that from Proposition 5.6, we recover the fact, already proved earlier, that $C$ is locally principal near $Q$ if and only if $\widetilde{\xi_{C}}$ goes not through $Q$. A minimal system of generators for the ideal of $C$ near $Q$ can now be easily determined by using the procedure suggested in [3] in the following way. Take a sequence of adjacent ideals (i.e. any two consecutive ideals have codimension one) as follows

$$
J_{0}=I H_{\mathcal{R}_{C}^{+}} \supsetneq J_{1} \supsetneq \ldots \supsetneq J_{m}=I_{Q} H_{\mathcal{R}_{C}^{+}}
$$


where

$$
m=1+\left[I_{Q}, H_{\mathcal{R}_{C}^{+}}\right]_{O}-\left[I, H_{\mathcal{R}_{C}^{+}}\right]_{O}
$$

Pick for $i=1, \ldots, m$, an element $g_{i}$ in $J_{i}$ and not in $J_{i+1}$. Then, the classes of $g_{0}, g_{1}, \ldots, g_{m-1}$ are a minimal system of generators of $I H_{\mathcal{R}_{C}^{+}} / I_{Q} H_{\mathcal{R}_{C}^{+}}$. Now, in the proof of Proposition 5.6, we have seen that $\mathcal{J}_{C}=(f I) \mathcal{H}_{\mathcal{R}_{C}}\left(\mathcal{L}_{I}\right)$. To obtain a minimal system of generators of $\mathcal{J}_{C}$ locally at $Q$ it is enough to consider

$$
\left(\frac{f g_{0}}{a t}, \frac{f g_{1}}{a t}, \ldots, \frac{f g_{m-1}}{a t}\right)
$$

where $a$ and $t$ are generic elements of $I$ and $H_{\mathcal{R}_{C}^{+}}$, respectively.

Example 5.7. Take $\eta: y=0$ in Example 3.7 and keep the notation used there. The curve $C=\widetilde{\eta}$ is not locally principal near $Q$ as the cluster $\mathcal{R}_{C}$ has positive excess at $p_{1}$. We have $I_{Q}=\mathfrak{m}^{2}$ and that the classes of $\left\{x^{2}-x y, x y\right\}$ generate $I H_{R_{+}} / I_{Q} H_{\mathcal{R}_{C}^{+}}$. Notice that $H_{\mathcal{R}_{C}^{-}}=I$. Thus, we can take $a=t=x$ as a generic element of $I$ and also of $H_{\mathcal{R}_{C}^{-}}$. Applying the procedure explained above, we have that $\left\{y(x-y) / x, y^{2} / x\right\}$ is a minimal system of generators of $\mathcal{J}_{C}$ near $Q$.

\section{Acknowledgements}

I wish to thank Eduard Casas-Alvero for helpful conversations on this topic. I also want to thank the anonymous reviewer for his kind comments and suggestions.

This research has been partially supported by the Spanish Committee for Science and Technology (DGYCIT), projects MTM2005-01518 and MTM2006-14234-C02-02, and the Catalan Research Commission.

\section{References}

[1] M. Alberich-Carramiñana and J. Fernández-Sánchez, Equisingularity classes of birational projections of normal singularities to a plane, Adv. in Math. 216 (2007) 753-770.

[2] M. Artin, On isolated rational singularities of surfaces, Amer. J. Math. 88 (1966) 129-136.

[3] E. Casas-Alvero, Filtrations by complete ideals and applications, Collect. Math. 49 (1998), no. $2-3,265-272$.

[4] Singularities of plane curves, Vol. 276 of London Mathematical Society Lecture Note Series, Cambridge University Press, Cambridge (2000).

[5] V. Cossart, O. Piltant, and A. J. Reguera-López, On isomorphisms of blowing-ups of complete ideals of a rational surface singularity, Manuscripta Math. 98 (1999), no. 1, 65-73.

[6] T. de Jong and D. van Straten, Deformation theory of sandwiched singularities, Duke Math. J. 95 (1998), no. 3, 451-522.

[7] P. Du Val, The unloading problem for plane curves, Amer. J. Math. 62 (1940) 307-311.

[8] F. Enriques and O. Chisini, Lezioni sulla teoria geometrica delle equazioni e delle funzioni algebriche., Collana di Matematica, N. Zanichelli,, Bologna (1915).

[9] J. Fernández-Sánchez, On sandwiched singularities and complete ideals, J. Pure Appl. Algebra 185 (2003) 165-175.

[10] T. S. Gustavsen, Equisingular deformations of sandwiched singularities, Compositio Math. 138 (2003) 233-244

[11] R. Hartshorne, Algebraic Geometry, Vol. 52 of Graduate Texts in Mathematics, Springer Verlag (1977).

[12] C. Huneke and J. D. Sally, Birational extensions in dimension two and integrally closed ideals, J. Algebra 115 (1988), no. 2, 481-500. 
[13] M. Lejeune-Jalabert and A. J. Reguera-López, Arcs and wedges on sandwiched surface singularities, Amer. J. Math. 121 (1999), no. 6, 1191-1213.

[14] J. Lipman, Rational singularities, with applications to algebraic surfaces and unique factorization, Inst. Hautes Études Sci. Publ. Math. (1969), no. 36, 195-279.

[15] D. Mumford, The topology of normal singularities of an algebraic surface and a criterion for simplicity, Inst. Hautes Études Sci. Publ. Math. (1961), no. 9, 5-22.

[16] A. Reguera-López, A curve selection lemma in spaces of arcs and the image of the Nash map, Compos. Math. 142 (2006), no. 1, 119-130.

[17] M. Spivakovsky, Sandwiched singularities and desingularization of surfaces by normalized Nash transformations, Ann. of Math. (2) 131 (1990), no. 3, 411-491.

Departament de Matemàtica Aplicada I, Universitat Politècnica de Catalunya, Av. Diagonal 647, 08028-Barcelona, Spain.

E-mail address: jesus.fernandez.sanchez@upc.edu 